\title{
Bone mineral status after pediatric spinal cord injury
}

\author{
M Kannisto ${ }^{1}$, H Alaranta ${ }^{2}$, J Merikanto ${ }^{1}$, H Kröger $^{3}$ and J Kärkkäinen ${ }^{4}$ \\ ${ }^{1}$ Children's Hospital, University of Helsinki; ${ }^{2}$ The Käpylä Rehabilitation Centre, ${ }^{3}$ Department of Surgery, Kuopio \\ University Hospital, Kuopio and ${ }^{4}$ Peijas Hospital, Vantaa, Finland
}

\begin{abstract}
The impact of spinal cord injury (SCI) on later bone mineral status was studied in 35 adults who had sustained their injury in childhood. The median age of the patients was 31 years, the median age at injury 12.9 years and the median time period from injury was 19 years. The methods used in the study were clinical interview and examination, measurement of bone mineral density (BMD) of the lumbar spine and the proximal femur with dual energy X-ray absorptiometry (DEXA) and estimation of bone turnover with biochemical markers. The densitometric examination revealed that the BMD at the lumbar spine was within the normal range but grossly decreased in the femoral region. Moreover, there was a significant difference in BMD between patients with high (C2-T6) and low (below T6) lesions in the lumbar spine as well as in the femoral region. Patients with lower lesions had higher BMD values. The markers of bone turnover which were studied were serum and urinary calcium and phosphate, serum alkaline phosphatase and its isoenzymes, osteocalcin, carboxyterminal propeptide of human type I procollagen (PICP), carboxyterminal telopeptide of type I collagen (ICTP) and urinary deoxypyridinoline. These markers of bone metabolism showed no signs of ongoing accelerated bone formation or resorption. The present study suggests that caution should be observed in weight bearing training or mobilisation of patients with pediatric SCI or perhaps with long standing SCI because of increased fracture risk. The prevention of dissociated osteoporosis should be investigated further in order to avoid fractures of weakened bones. The modes of prevention might be found in the use of modern pharmacotherapy of osteoporosis and from correctly dosaged physical training.
\end{abstract}

Keywords: pediatric spinal cord injury; osteoporosis; bone density; spinal cord injury; bone metabolism

\section{Introduction}

Spinal cord injury (SCI) is known to lead to profound perturbation of bone metabolism which results in dissociated demineralisation of central and peripheric bone. $^{1,2}$

A new steady state between formation and resorption of bone is reported to occur within 6 months to 2 years after the injury. ${ }^{3}$

Attemps to restore lost bone mineral density (BMD) have been unsuccessful which has led to a focus on prophylactic methods in preserving the BMD. Several studies have not shown an increase in BMD resulting from training programs with mechanical orthosis, with prolonged training sessions or with electrically induced ergometry training). ${ }^{1,4-8}$ However, there has been shown to be a positive influence on BMD caused by regularly performed passive standing as well as electrically induced cycle training of paralysed limbs and this has also been reported to have a beneficial effect on the preserva-

Correspondence: M Kannisto, Department of Physiatry, Peijas Hospital, Sairaalakatu 1, Vantaa 01400, Finland tion of bone mass in osteoporosis found in paraplegics. 9,10

Antiresorptive compounds such as bisphosphonates are promising agents for reducing bone loss in immobilisation osteoporosis, although treatment modalities are not yet established in humans. ${ }^{11-14}$

During growth the trabecular bone of the skeleton matures to its peak density at around 16-20 years of age and then begins to decline gradually. ${ }^{15,16}$ The accrual of periosteal cortical bone, on the contrary, continues during life and leads to an increase in the diameter of the shaft of long bones. ${ }^{17}$

SCI in an adolescent, whose underlying rate of bone turnover is high, leads to bone mineral loss caused by immobilisation during the healing of the fracture and afterwards by the reduced activity and unloading of the paralysed limbs and trunk. In young SCI patients, during the acute phase the calcium balance becomes negative and can lead to serious immobilisation hypercalcemia. ${ }^{18}$ Later on, disproportioned growth of legs (hypotrophy), secondary scoliosis, fractures and development of 
heterotopic ossification are other known adverse effects of SCI in children. In the general population the main shortcomings related to osteoporosis are increased risk of fractures and bone pain.

In an adolescent SCI-patient the development of immobilisation osteoporosis interrupts the normal process of accelerated bone formation connected to growth. The aim of the present study was to investigate the later bone mineral status of those patients who had sustained SCI in childhood and, especially, to study the question of whether bone formation and resorption is equalised years after the initial derangement of bone metabolism.

\section{Patients}

Ten females and 25 males who had sustained SCI in childhood participated in this study. All the patients were interviewed and clinically examined. The spinal lesions were classified according to the level and the completeness of the injury in accordance with the standards of neurological classification of spinal cord injury patients. ${ }^{19}$ Twenty-four patients in the study group had complete paraplegia. Three patients had incomplete paraplegia, three patients were tetraplegics and five patients had incomplete tetraplegia (Table 1).

At the time of the study the median age of the patients was 31 years (range 18-63). The median age at injury was 12.9 years (range from birth to 17.1 years) and the median time period from the injury was 19 years (range 1.5-57). The results of the laboratory test of one of the subjects was excluded from the study because of profoundly altered blood chemistry and the lack of a valid urinary sample. The changes of blood chemistry were related to chronic alcohol abuse and to a large decubitus with underlying osteitis. The densitometry of the lumbar column and hip of the same subject failed because of extreme scoliosis (Cobb > 110 degrees) and advanced osteoporosis of the luxated hips. Thus the final group consisted of 34 patients.

This study has been approved by the ethical committee of the Children's Hospital, University of Helsinki, Finland. Informed consent was obtained from the participants.

Table 1 Classification of patients according to the level of the lesion

\begin{tabular}{llcc}
\hline & & $\begin{array}{c}\text { Complete } \\
\text { ASIA A }\end{array}$ & $\begin{array}{c}\text { Incomplete } \\
\text { ASIA BCD }\end{array}$ \\
\hline Tetraplegia & high (C2-5) & 2 & 3 \\
& intermediate (C6) & 1 & 2 \\
Paraplegia & high (T1-T6) & 7 & 1 \\
& low (T7 or below) & 17 & 2 \\
Total & & 27 & 8 \\
\hline
\end{tabular}

\section{Methods}

\section{Densitometry}

Hologic QDR-1000 X-ray bone absorptiometer with software version 6.10 (Hologic, Inc., Waltham, USA) was used for BMD measurements taken from the lumbar spine (L2-L4), the proximal femur and from specific sites at the proximal femur region (the femoral neck, trochanteric area, intertrochanteric area, Ward's triangle). The areas and calculations of the regions measured were obtained automatically by the instrument's computer program.

The definition for the term osteoporosis adopted for this study was a decrease of BMD of more than 2.5 SD compared to peak bone density reference data obtained from a Caucasian population (T-score). The bone density reference data was provided by the manufacturer of Hologic QDR systems. ${ }^{20}$

It was possible to measure the BMD of the lumbar spine in 29 of the 35 patients. Spondylodesis and extreme scoliosis obstructed the analysis of the BMD from the lumbar spine in five cases. In one of the cases the degenerative changes of the lumbar spine formed an obstacle to the analysis.

The BMD of the hip was measured in 27 hips. The reason for the failure of the equipment to measure the BMD in eight cases was the unsufficient contrast between mineralised and non-mineralised tissue.

\section{Laboratory assays of bone metabolism}

\section{Blood and urine samples}

Fasting blood samples were obtained from all participants between the hours of 8 to 10 a.m. Serum and urine samples were stored at $-20^{\circ} \mathrm{C}$ until assayed. The patients were instructed to collect their urine starting at 8 a.m. and to continue for $24 \mathrm{~h}$ until the next morning. To eliminate interassay variation, urinary deoxypyridinoline (DPD), carboxyterminal propeptide of human type I procollagen (PICP) and the carboxyterminal telopeptide of type I collagen (ICTP) were assayed in a single session.

\section{Urinary calcium, phosphate and creatinine}

Urine concentrations of ionised calcium and phosphate were measured with standard methods. Serum and urinary concentrations of creatinine were measured by a modified Jaffe method.

\section{Alkaline phosphatase (AP) and its bone isoenzyme (BAP)}

The serum activity of AP and especially its bone isoenzyme is high during childhood and also afterwards in conditions of increased bone formation. Isoenzymes of AP derived from liver and bone were measured separately in 17 cases out of 34 . Total serum AP was measured with the Scandinavia method (SCE) 
and polyacylamide gel electrophoresis was used to measure its isoenzymes.

\section{Osteocalcin (OC) assay}

$\mathrm{OC}$ or bone Gla protein is a non-collagenous vitamin $\mathrm{K}$-dependent protein that is specific for bone tissue and dentin. It is synthesised by osteoblasts and incorporated into the extracellular matrix of bone. ${ }^{21}$

Serum OC correlates with histomorphometric parameters of bone formation and it is a marker of bone turnover when resorption and bone formation are coupled. Serum OC was measured by radioimmunoassay. ${ }^{22}$

\section{Urinary hydroxyproline (HYP)}

Urinary HYP reflects the resorption rate of bone although $50 \%$ is from non-bone sources. Urinary HYP was determined with spectrophotometry. ${ }^{23}$

\section{Urinary Deoxypyridinoline (DPD)}

Pyridinolines are non-reducible cross-links that stabilise the collagen chains within the extracellular matrix. ${ }^{24}$ They are specific for bone collagen and are released from bone matrix during its degradation by osteoclasts and excreted in the urine. Urinary DPD was measured with a commercial competitive enzyme immunoassay made by United Laboratories Ltd, Helsinki; the reagent manufacturer was Metra Biosystems, Inc., Palo Alto, California. The results obtained were corrected for the urine concentration with the creatinine concentration of the sample.

The carboxyterminal propeptide of human type I procollagen (PICP) and cross-linked carboxyterminal telopeptide of type I (ICTP).

Serum PICP has been shown to be a marker of bone formation and serum ICTP a marker of bone resorption. $^{25}$ Together with PICP, ICTP offers a possibility of estimating the balance between type I collagen formation and degradation. PICP and ICTP were measured with a standard kit of radioimmunoassay provided by Orion Diagnostica, Finland. ${ }^{26,27}$

\section{Statistical methods}

The D-BASE III plus (Ashton-Tate) computer program was used to store and process the data. The statistical analyses were carried out using Statgraphics software (Statistical Graphics Corp). A paired onetailed Student's $t$-test for dependent samples was used to compare the BMD of the lumbar spines and hips. Comparisons of BMD at various sites between the subgroup means of the sample of patients was performed using a one-way analysis of variance (ANOVA). Post-hoc intercomparison of the subgroup means was performed using Tukey's test.

A multiple regression was used to analyze the influences of body weight, height, age at injury, time from injury and age at the time of examination to the BMD of the lumbar spine, the proximal femur and the femoral neck.

\section{Results}

Bone mineral density (BMD)

In the cases measured the BMD levels were within the normal range in the lumbar spine (Table 2). The mean BMD at the lumbar spine was $1.08 \mathrm{~g} / \mathrm{cm}^{2}$ (range $0.84-1.54, \mathrm{SD} 0.17)$. This value represents $99.5 \%$ of the age and sex adjusted mean (Z-score) and $-0.04 \mathrm{SD}$ of peak bone mass measured in 30-year old persons of the same gender as the patients ( $\mathrm{T}$ score) (Tables 2 and 3).

At the hips, accurate subtraction between bone and soft tissues with the densitometer failed in seven out of the 34 patients. The BMD at the proximal femur was on an average $0.72 \mathrm{~g} / \mathrm{cm}^{2}$ (range $0.39-1.46$, SD 0.23 ) which is $-2.05 \mathrm{SD}$ of the age and gender adjusted mean value ( $Z$-score). The mean $\mathrm{T}$-score was -2.61 and thus represents established osteoporosis.

Table 2 Bone mineral density $\left(\mathrm{gm} / \mathrm{cm}^{2}\right)$ in different types of SCI

\begin{tabular}{lccrc}
\hline & Mean & $S D$ & $\mathrm{n}$ & Z-score \\
\hline Lumbar spine & & & & \\
Complete paraplegia & 1.125 & 0.180 & 20 & 0.52 \\
Incomplete paraplegia & 1.205 & 0.000 & 1 & 1.45 \\
Complete tetraplegia & 1.005 & 0.010 & 3 & -0.64 \\
Incomplete tetraplegia & 0.896 & 0.048 & 5 & -1.77 \\
Entire sample & 1.077 & 0.173 & 29 & 0.002 \\
Proximal femur & & & & \\
Complete paraplegia & 0.753 & 0.272 & 17 & -1.56 \\
Incomplete paraplegia & 0.733 & 0.197 & 2 & -2.11 \\
Complete tetraplegia & 0.621 & 0.129 & 3 & -3.19 \\
Incomplete tetraplegia & 0.687 & 0.128 & 5 & -2.74 \\
Entire sample & 0.724 & 0.230 & 27 & -2.05 \\
\hline
\end{tabular}

Table 3 Bone mineral density of the patients compared to young adults

\begin{tabular}{lrcr}
\hline & $\%$ & $T$-score (30) & \multicolumn{1}{c}{$\mathrm{n}$} \\
\hline Lumbar spine & & & \\
Complete paraplegia & 105 & 0.41 & 18 \\
Incomplete paraplegia & 115 & 1.44 & 1 \\
Complete tetraplegia & 93 & -0.65 & 3 \\
Incomplete tetraplegia & 82 & -1.77 & 5 \\
Entire sample & 100 & -0.04 & 27 \\
Proximal femur & & & \\
Complete paraplegia & 80 & -2.42 & 14 \\
Incomplete paraplegia & 74 & -2.27 & 2 \\
Complete tetraplegia & 60 & -3.32 & 3 \\
Incomplete tetraplegia & 65 & -2.96 & 5 \\
Entire sample & 74 & -2.61 & 24 \\
\hline
\end{tabular}


The BMD in the femoral neck was $0.69 \mathrm{~g} / \mathrm{cm}^{2}$ (range $0.38-1.38$ SD 0.19). In Ward's triangle the mineral density was $0.60 \mathrm{~g} / \mathrm{cm}^{2}$ (range $0.22-1.45$, SD 0.24). The lowest measurements were at the intertrochanteric level where BMD was on an average $0.52 \mathrm{~g} / \mathrm{cm}^{2}$, ranging from 0.24 to 0.94 .

At the lumbar spine 10/29 of the patients had a Tscore which was under $-1 \mathrm{SD}$ and $3 / 29$ of the patients had T-scores less than $-2 \mathrm{SD}$. None of the patients had a $\mathrm{T}$-score less than $-2.5 \mathrm{SD}$ at the lumbar spine. At the femoral neck $21 / 27$ of the patients had T-scores under $-1 \mathrm{SD}, 19 / 27$ had T-scores less than $-2 \mathrm{SD}$ and $16 / 27$ had a $\mathrm{T}$-score less than $-2.5 \mathrm{SD}$.

\section{The dissociation between axial and peripheral BMD}

(lumbar spine vs total femoral area) was statistically significant $(t=7.36, \mathrm{df}=25, P<0.001)$.

The results of ANOVA showed that at the lumbar level there was a statistical difference in BMD between tetraparetics $(n=5)$ who had lower BMD values than paraplegics $(n=20)$, paraparetics $(n=1)$ and tetraplegics $n=3((\mathrm{df}(3,25), F=3.2387, P<0.04))$ although the post-hoc intercomparison showed no essential difference between the subgroups (Table 3 ).

At the hip level ANOVA showed no evidence of difference between the subgroups.

The sample was divided into two groups in order to study the influence of lesion level on BMD at lumbar levels and in the proximal femur region. The group A $(n=14)$ consisted of $\mathrm{C} 1-\mathrm{T} 6$ lesions and group B $(n=15)$ of lesions at and below T7. The mean BMD in group A at lumbar level was $0.98 \mathrm{~g} / \mathrm{cm}^{2}$ (SD 0.12, range $0.84-1.32$ ) and $1.16 \mathrm{~g} / \mathrm{cm}^{2}$ (SD 0.18, range $0.86-1.54)$. In group $\mathrm{B}$, at the proximal femur region, the corresponding results were $0.61 \mathrm{~g} / \mathrm{cm}^{2}$ (SD 0.14, range $0.39-0.89$ ) and $0.83 \mathrm{~g} / \mathrm{cm}^{2}$ (SD 0.24, range $0.57-1.46)$. These differences between groups $\mathrm{A}$ and $B$ were also statistically significant at lumbar $((\mathrm{df}(1,27), F=9.90079 . \quad P<0.004))$ and hip $((\mathrm{df}(1,25)$, $F=7.2388, P<0.01))$ levels.

Multiple regression analysis showed that BMD of the proximal femur $(\beta=0.49, P \geqslant 0.01)$ and the femoral neck $(\beta=0.57, P<0.01)$ correlated to bodyweight but not to body height, age at the time of injury, age at the time of examination or to the time elapsed since injury.

\section{Biochemical estimation of turnover}

Biochemical markers of bone metabolism showed no signs of still ongoing accelerated bone formation or resorption (Table 4). No indications of therapeutic or other interventions could be predicted with indices of biochemical activity.

\section{Discussion}

In the present study a clear dissociation of BMD was found between lumbar spine and hip levels in pediatric
Table 4 Biochemical markers of bone turnover

\section{Mean $S D \quad n \quad$ Reference range}

\begin{tabular}{|c|c|c|c|c|}
\hline \multicolumn{5}{|c|}{ Formation } \\
\hline \multicolumn{5}{|c|}{ Serum alkaline phosphatase } \\
\hline Total & 170 & 86 & 32 & $60-275 \mathrm{u} / 1$ \\
\hline Bone isoenzyme & 68 & 33 & 16 & $<110 \mathrm{u} / 1$ \\
\hline \multicolumn{5}{|l|}{ Serum osteocalcin } \\
\hline Males & 7.2 & 5.2 & 23 & $4.0-8.0 \mathrm{ug} / 1$ \\
\hline Females & 3.8 & 2.5 & 10 & $2.0-6.0 \mathrm{ug} / 1$ \\
\hline Serum PICP & 126 & 38 & 33 & $50-170 \mathrm{ug} / \mathrm{l}$ \\
\hline \multicolumn{5}{|l|}{ Resorption } \\
\hline Serum ICTP & 4.4 & 2.5 & 33 & $1.5-4.5 \mathrm{ug} / 1$ \\
\hline $\begin{array}{l}\text { Fasting urinary } \\
\text { calcium }\end{array}$ & 2.95 & 1.7 & 25 & $\begin{array}{c}>18 \text { years } 1.25- \\
5.50 \mathrm{mmol} / 1\end{array}$ \\
\hline \multicolumn{5}{|l|}{ Urinary hydroxyproline } \\
\hline $18-20$ years & 0.28 & 0.2 & 4 & $\begin{array}{c}0.1-0.22 \mathrm{mmol} / \\
24 \mathrm{~h} / \mathrm{M} 2\end{array}$ \\
\hline $21-65$ years & 0.12 & 0.11 & 22 & $\begin{array}{c}0.07-0.18 \mathrm{mmol} / \\
24 \mathrm{~h} / \mathrm{M} 2\end{array}$ \\
\hline \multicolumn{5}{|c|}{ Urinary deoxypyridinoline } \\
\hline Males $20-50$ years & 5.8 & $\begin{array}{c}1.8 \\
\mathrm{nmol} /\end{array}$ & $\begin{array}{c}19 \\
\text { nmol }\end{array}$ & $\begin{array}{l}1.0-8.0 \\
\text { creatinine }\end{array}$ \\
\hline Females $20-50$ years & 8.3 & $\begin{array}{c}5.5 \\
\mathrm{nmol} /\end{array}$ & $\begin{array}{c}9 \\
\text { nmol }\end{array}$ & $\begin{array}{c}2.0-9.0 \\
\text { creatinine }\end{array}$ \\
\hline
\end{tabular}

SCI patients years after the injury. The BMD values were within the normal range at the lumbar spine but indicated serious osteoporosis at hip level. In eight out of 35 cases the equipment failed to measure the BMD due to insufficient contrast between the femur and surrounding soft tissues. In these cases the BMD values were probably very low but are not seen in sample means and in the comparisons.

These results are in agreement with other studies which confirm the dissociation of mineral status between peripheral and axial bone after an acute phase of paraplegia. 1,2

Bone mineral density is highly correlated with bone strength. ${ }^{28}$ The risk of osteoporotic hip fracture is not quite the same for the general population and for paralysed persons because there is less weight bearing at hip level in a sitting position than when standing. However, advanced osteoporosis and augmented fracture risk has to be taken into consideration when standing is used as rehabilitation means in chronic and possibly spastic SCI persons.

Axial weight loading through sitting in a wheelchair has been used to explain the confinement of the BMD deficit to the paralysed extremities. ${ }^{1,2,29}$ In animal experiments the skeletal mass has been consistently shown to depend on skeletal loading. ${ }^{30}$ The bone mineral loss is also confined to neurologically affected upper limbs in tetraplegics, although it is less pronounced. ${ }^{29,31}$ No exact aetiology of this diminution of bone mass seems 
to be known although reduced muscle mass and motor activity as well as altered circulation may be factors of importance.

The present study showed that the BMD at the proximal femur and in the femoral neck was connected with body weight but not with body height, age at time of injury, age at examination nor with the time elapsed since injury. A similar correlation was not found for the BMD of the lumbar spine. Such a decline of the BMD in the femoral area may be in related to the smaller degree of weight loading on the legs, which depends of the body weight and the type of spinal lesion.

An increase in BMD after immobilisation for reasons other than paralysis has been shown to require prolonged and rigorous training. Even then, bone mass increases by only a few percent, although the reported increase in strength can be as high as $60 \% .^{32}$ Daily and long-term loading of the axial skeleton maintained by sitting in a wheelchair can fulfill skeletal loading criteria for maintaining the BMD, which is not true for inferior extremities. Despite these demands for intense loading training in order to maintain bone mass, there is also scientific evidence that lesser training can be useful. In a study by Goemare et $a l^{9}{ }^{9}$ the BMD of the femoral shaft was higher in paraplegic patients who performed passive weight bearing than in patients who did not. ${ }^{9}$ In healthy young persons peak bone mass is a major determinant of bone mass later in life, but regular exercise also has an influence on BMD. In a study of by Välimäki et al. ${ }^{33}$ on the determinants of peak bone mass, the authors showed that BMD at the femoral neck was $7.6-10.5 \%$ higher in subjects with the most regular exercise compared with those with the least exercise. $^{31}$

The initial resorption of bone by osteoclastic activity which dominates over osteoblastic activity in SCI patients has been reported to have a time limit. In a histomorphometric study the reduction in the amount of trabecular bone averaged from $8 \%$ to $33 \%$ over 6 months before a new steady state was reached in paraplegic patients. ${ }^{3}$ Osteoclastic activity returned to normal at about 40 weeks while the bone volume remained constant after the 25 th week. ${ }^{34}$ On the other hand an increase of osteopenia in the paralysed limbs with time after the injury has also been reported. $^{29}$

In our study the T-scores showed the presence of osteoporosis of the peripheral bone (Table 3). In the adolescent SCI-patients the amount of remineralisation of the axial bone after the initial accident is not known. Without successful, and to date still not evidence-based prevention, the peripheral bone mass seems to remain grossly reduced.

The BMD in the lumbar and hip regions correlated to the level of neural lesion. One possible explanation for this is the advanced mobility and more extended use of musculature amongst those with low lesions when compared to those with higher lesions. The possible role of spastic/flaccid conditions could not be discriminated in this study because of the small number of flaccid lesions. Although the lesion level was an important determinant of BMD there was not a statistically significant difference between patients with complete and incomplete neural lesions.

The actual dynamic state of bone metabolism was assessed with biochemical markers which reflect whole skeletal bone formation and resorption rates. The markers of bone formation (BAP, OC, PICP) as well as the markers of bone resorption (HYP, DPD, ICTP) used in this study were within reference values. The clinical performances of urinary HYP and DPD measurements are comparable in the case of large changes in bone resorption. For modest changes urinary DPD is more accurate and has a higher discrimination power than urinary HYP. ${ }^{35}$ The measurement of cross-linked carboxyterminal telopeptide of type I collagen (ICTP) in serum has been suggested as a marker of bone resorption. ${ }^{25}$ Together with PICP, ICTP offers a possibility for estimating the balance between type I collagen formation and degradation. In a steady-state situation the levels of these two peptides should show a change in the same direction. $^{25}$

It has been reported that the development of bone mass in patients with pediatric SCI never reaches normal levels, which exposes these patients to fractures. 1,36 In the present study the BMD in the lumbar spine reached the level of the general reference population, which shows that at least the mineralisation of central bone returns to normal levels in adolescents with pediatric SCI after initial phases of SCI. The amount of bone mineral loss at hip level was shown to remain at alarmingly low values. This exposes these patients to below threshold values of osteoporotic fractures with all the consequences associated with delayed healing and prolonged immobilisation.

Our results suggest that there is a need to develop pharmacological and physical modalities in order to prevent bone mineral loss in pediatric SCI-patients during the initial weeks and possible months of immobilisation, and secondarily to discover what are reasonable treatments for avoiding mineral loss in peripheral bone.

\section{References}

1 Biering-Sørensen F, Bohr H, Schaadt O. Bone mineral content of the lumbar spine and lower extremities years after spinal cord lesion. Paraplegia 1988; 26: 293 - 301

2 Leslie W, Nance P. Dissociated hip and spine demineralization: a specific finding in spinal cord injury. Arch Phys Med Rehabil 1993; 74: $960-964$

3 Minaire $\mathrm{P}$ et al. Quantitative histological data on disuse osteoporosis: comparison with biological data. Calcif Tissue Res 1974; 17: $53-73$.

4 Ogilvie C, Bowker P, Rowley DI. The physiological benefit of paraplegic orthotically aided walking. Paraplegia 1993; 31: 111 115.

5 Thoumie $\mathrm{P}$ et al. Restoration of functional gait with the RGO-II hybrid orthosis. Paraplegia 1995; 33: 654-659. 
22 Nichol's Human Oc RIA, Nichol's Institute Diagn., San Juan Capistrano, CA, USA.

23 Kivirikko KI, Laitinen O, Prockop DJ. Modifications of a specific assay for hydroxyproline in urine. Anal Biochem 1967; 19: $249-55$.

24 Eyre DR. Collagen crosslinking amino-acids. Meth Enzymol 1987; 144: $115-139$.

25 Eriksen E et al. Serum markers of type I collagen formation and degradation in metabolic bone disease. Correlation to bone histo-morphometry. J Bone Miner Res 1993; 8: 127-132.

26 Melkko J, Niemi S, Risteli L, Risteli J. Radioimmunoassay of the carboxyterminal propeptide of human type I procollagen. Clin Chem 1990; 36: $1328-1332$.

27 Risteli $\mathbf{J}$ et al. Radioimmunoassay for the pyridinoline crosslinked carboxyterminal telopeptide of type I collagen: A new serum marker of bone collagen degradation. Clin Chem 1993; 39: $635-640$.

28 Hayes WC, Gerhart TN. Biomechanics of bone. Applications for assessment of bone strength. In: Peck W (ed). Bone and Mineral Research/3. Elsevier Science Publishers V.B: Amsterdam 1985; pp 259-294.

29 Finsen V, Indredavik B, Fougner K. Bone mineral and hormone status in paraplegics. Paraplegia 1992; 30: $343-347$.

30 Rubin CT, Lanyon LE. Regulation of bone mass by mechanical strain magnitude. Calcif Tissue Int 1985; 37: 411-417.

31 Griffiths H, D'Orsi C, Zimmerman R. Use of I (125) photon scanning in the evaluation of bone density in a group of patients with spinal cord injury. Investigative Radiology 1972; 7: $107-$ 111.

32 Sievänen $\mathrm{H}$ et al. Bone mineral density and muscle strength of lower extremities after long-term strength training, subsequent knee ligament injury and rehabilitation: a unique 2-year followup of a 26-year-old female student. Bone 1994; 15: 85-90.

33 Välimäki M et al. Exercise, smoking, and calcium intake during adolescence and early adulthood as determinants of peak bone mass. BMJ 1994; 309: 230-235.

34 Minaire P. Immobilisation osteoporosis: A review. Clin Rheumatol 1989; 852: 95-103.

35 Bettica $\mathrm{P}$ et al. Bone-Resorption markers galactosyl hydroxylysine, pyridinium crosslinks, and hydroxyproline compared. Clin Chem 1992; 11: 2313-2318.

36 Nishiyama N, Kuwahara T, Matsuda I. Decreased bone density in severely handicapped children and adults, with reference to the influence of limited mobility and anticonvulsant medication. European Journal of Pediatrics 1986; 144: 457-463. 